\title{
Le ministère des Arts (1881-1882) ou l'institutionnalisation manquée d'une politique artistique républicaine
}

«Les grands services publics naissent d'un besoin social, plus ou moins grand, mais toujours existant au moins à la création du service. En voyant naître et disparaître les départements ministériels, en les voyant se transformer, nous assisterons, dans une certaine mesure, aux transformations sociales dont la France fut l'objet. $^{1}{ }_{\text {» }}$

Dans les tout derniers jours du second Empire, le ministère des Beaux-Arts qui remplaçait depuis peu celui de la Maison de l'Empereur est démantelé. Dès septembre 1870, ses services sont répartis entre les ministères des Travaux publics, de l'Instruction publique et des Cultes. À partir de cette date et tout au long de la IIIe République, le traitement gouvernemental des beaux-arts reste partiellement assuré par une simple direction ou un sous-secrétariat d'État (créé en 1875) aux attributions fluctuantes. Envisagées et débattues à de nombreuses reprises, la formation d'un nouveau ministère et, avec elle, la formalisation d'une politique artistique n'ont cependant jamais été réalisées ${ }^{2}$.

Cette absence n'était pas inéluctable : c'est ce que permet de rappeler la brève exception qui constitue l'objet de cet article. Le 14 novembre 1881, la création d'un ministère des Arts, confié au député et critique d'art Antonin Proust, inscrit en effet la politique artistique dans les fonctions d'un gouvernement républicain. Inscription toute temporaire, puisque ce ministère disparaît après deux mois et demi d'existence, le 29 janvier 1882, et n'est jamais reconstitué. Au-delà du récit d'une « expérience », revenir sur cet épisode permet d'éclairer la genèse de l'intervention culturelle publique, en exhumant une forme historique à un moment envisagée, puis écartée, du traitement public de l'art ${ }^{3}$. En plus de la définition d'une fonction gouvernementale nouvelle que marque la création d'un ministère ce "possible évacué » consiste de fait en la promotion d'une représentation spécifique de l'art et de ses problèmes. Cette dernière trouve son origine dans la promotion des arts dits "décoratifs" ou « industriels » engagée à partir du milieu du siècle en France. La double rupture qui la caractérise permet aux promoteurs de l'intervention publique de (re)penser et (re)légitimer le traitement étatique des questions artistiques : la remise en cause de l'opposition traditionnelle entre « le Beau et l'Utile » tend à démontrer les intérêts sociaux et économiques de l'art, et l'abandon de la catégorie élitiste des « beaux-arts » constituée sous l'Ancien régime au profit d'une appréhension unifiée des arts en général conduit à produire une définition du domaine artistique ajustée aux principes censés fonder l'action d'un gouvernement républicain. Édifié sur cette base, le ministère des Arts doit en retour faire correspondre les structures sociales et mentales de la vie artistique « à l'état de nos mœurs », comme le dit le ministre ${ }^{4}$.

1. A. Racinet, La spécialisation ministérielle en France, thèse de doctorat en Droit, Paris, Recueil J.-B. Sirey, 1910 , p. 8.

2. Pour une analyse des raisons de cette absence, on se permet de renvoyer à notre article, « L'art et l'État au début de la IIIe République, ou les conditions d'impossibilité de la mise en forme d'une politique ", Genèses, sciences sociales et histoire, 23, juin 1996, p. 6-29. Pour une histoire de l'administration des beaux-arts, voir aussi, dans une perspective différente, Marie-Claude Genet-Delacroix, Art et État sous la IIIe République. Le système des beaux-arts, 1870-1940, Paris, Publications de la Sorbonne, 1992.

3. Outre l'article précité, voir plus généralement notre ouvrage, La politique culturelle. Genèse d'une catégorie d'intervention publique, Paris, Belin, coll. Socio-histoires, 1999.

4. Antonin Proust, L'Art sous la République, Paris, Bibliothèque Charpentier, 1892, p. 26. 
La création de ce ministère peut ainsi tout particulièrement s'analyser dans une perspective durkheimienne comme un résultat des échanges entre «le corps social» et "l'organe gouvernemental » qui « se développe avec la division du travail, non pour y faire contrepoids, mais par une nécessité mécanique $»^{5}$. Une nouvelle division du travail s'esquisse, qui tend à relier les activités artistique et économique ; le ministère des Arts se fonde sur cette nouvelle organisation et les représentations qui y sont liées; il est en retour censé les faire advenir. Ces relations à double sens qui unissent l'administration gouvernementale aux structures sociales ne s'inscrivent cependant pas dans une évolution linéaire à l'issue inéluctable. Dans le cas du ministère des Arts comme dans bien d'autres, elles sont aussi marquées par des hésitations, des conjonctures singulières, voire des retours en arrière ${ }^{6}$. En l'occurrence, les échanges et l'innovation institutionnelle à laquelle ils conduisent n'avaient rien d'évident. On verra en effet qu'ils ont nécessité la mobilisation conjointe d'une fraction particulière du champ artistique et de réformateurs gouvernementaux. Il nous faudra alors rappeler les conditions politiques et institutionnelles exceptionnelles qui ont permis aux ressources des réformateurs d'être pleinement employées, et à leur projets d'être réalisés. Ces conditions de possibilité conjoncturelles ont en retour largement contribué à dessiner les formes de cette institution, et l'orientation de la politique élaborée en son sein. Elles expliquent aussi — pour partie au moins - son échec à s'établir durablement dans les catégories de pensée et d'action gouvernementales ${ }^{7}$

\section{Des alliances entre artistes et politiques}

« Jamais peut être on n'a autant parlé de beaux-arts en France que durant l'année 1881. » 8

Au début des années 1880 , les producteurs culturels ne se définissent pas encore comme des « intellectuels », c'est-à-dire entre autres par leur distance à l'égard de la politique officielle 9 . Des savants, artistes et hommes de lettres apportent leur soutien au régime républicain en cours d'installation. Ils entretiennent des relations étroites avec le personnel parlementaire et

5. Émile Durkheim, De la division du travail social, Paris, PUF, 1983 (1930), p. 350. Voir aussi ibid., p. 197 205.

6. Pour un exemple - sur une autre période et sur un autre objet - d'une analyse en ces termes voir, au sujet des effets de conjoncture sur les créations institutionnelles, Didier Georgakakis, « Différenciation administrative et conjonctures critiques : la création du Commissariat Général à l'Information et ses avatars (juillet 1939-avril 1940) », in Martine Kaluszinski, Sophie Wahnich (dir.), L'État contre la politique ?, Paris, L'Harmattan, p. 303320 ; sur les échecs de la spécialisation administrative, du même auteur, Information et propagande d'État sous la IIle République, thèse, Institut d'études politiques de Lyon, 1995, p. 61 et suivantes. Sur l'intérêt d'une analyse des échecs pour la sociologie historique, voir plus généralement Charles Tilly, Roads from Past to Future (Legacies of Social Thought), New York, Rowman \& Littlefield Publishers, 1997.

7. Cet article repose sur quatre principaux types de sources: des documents officiels (rapports et débats parlementaires) ; un corpus d'ouvrages sur les rapports entre art et État (mémoires de ministres et fonctionnaires, essais); le dépouillement systématique des principales revues d'art (années 1881 et 1882); des articles de presse, sélectionnés pour l'essentiel à partir des tables du Temps. On a par ailleurs consulté les principaux dictionnaires biographiques suivants: Emile Bellier de la Chavignerie, Dictionnaire général des artistes de l'école française, vol. 1, Paris, Renouard, 1882; Adolphe Bitard, Dictionnaire générale de biographie contemporaine, Paris, Vanier, 1880 ; Alfred Dantès, Dictionnaire biographique et bibliographique des hommes les plus remarquables dans les lettres, les sciences et les arts, Paris, Boyer et Cie, 1875 ; Dictionnaire de biographie française, Paris, Letouzey, 1956 ; Ernest Glaeser, Biographie nationale des contemporains, Paris, Glaeser, 1878 ; Louis-Gustave Vapereau, Dictionnaire universel des contemporains, Paris, Hachette, 1893. Je tiens à remercier Yves Déloye, Didier Georgakakis, Frédérique Matonti et Didier Renard pour leurs remarques sur une version antérieure de ce texte.

8. Victor Champier, L'année artistique 1881-1882, Paris, A. Quantin, 1882, p. 1.

9. Christophe Charle, Naissance des « intellectuels », Paris, Minuit, 1990. 
gouvernemental ${ }^{10}$. Dans le domaine artistique, ces échanges se concrétisent notamment par la réforme du Salon annuel des beaux-arts, dont l'édition de 1881 est pour la première fois organisée par un comité d'artistes, la Société des artistes français, ce qui permet une moindre emprise de la tutelle administrative et académique. Se dessine alors une correspondance entre les concurrences internes au champ artistique et le clivage structurant le champ politique, les artistes « indépendants » étant soutenus par des républicains contre les conservateurs en art et en politique ${ }^{11}$. Dans cette configuration favorable des relations entre les champs artistique et politique, une mobilisation pour le développement de l'intervention gouvernementale émerge des marges du champ artistique : le mouvement composite des arts décoratifs et industriels forme le point d'ancrage d'une " campagne » organisée dans le courant de l'année 1881 pour la création d'un ministère spécifiquement consacré à l'art.

\section{La « nébuleuse réformatrice » de l'art industriel 12}

Le mouvement des arts " décoratifs » et-ou " industriels » émerge en Europe au milieu du XIXe siècle ${ }^{13}$. L'exposition universelle de Londres en 1851 révèle l'intensité des concurrences que se livrent les pays européens dans ce domaine. La création d'une importante institution destinée à soutenir les progrès des arts industriels britanniques, le South Kensington Museum, fait craindre que la France ne se laisse distancer. Dès le début des années 1850, des rapports officiels, comme ceux du comte Léon de Laborde, archéologue et membre de l'Académie des inscriptions, mettent en garde contre la «menace » qui pèse sur la "supériorité française », et préconisent en conséquence la création d'un musée des arts industriels, d'une école centrale des arts appliqués, ou encore l'organisation d'expositions temporaires. C'est en ce sens qu'en 1864 est créée l'Union des beaux-arts appliqués à l'industrie. Dans ce regroupement hétérogène d'artisans d'art, d'industriels, de collectionneurs, d'amateurs, d'archivistes, d'administrateurs, d'historiens d'art, etc., les nostalgiques de la décoration du XVIIIe siècle côtoient les réformateurs qui tendent à briser la distinction hiérarchique des arts libéraux et des arts mécaniques cristallisée dans la catégorie des beaux-arts ${ }^{14}$. Les mots d'ordre communs - réunir le Beau et l'Utile, industrialiser l'art, rendre l'industrie plus artistique - rassemblent des finalités diverses, à la fois commerciales - développer l'exportation des produits de l'industrie nationale - «démocratiques » rendre le « Beau » accessible à tous — et moralisatrices — « améliorer le goût public » ${ }^{15}$.

10. Pour une étude des rapports entre artistes et politiques à cette période, $\mathrm{Cf}$. Patricia Mainardi, The End of the Salon. Art and the State in the Early Third Republic, Cambridge, Cambridge University Press, 1993.

11. Voir à ce propos Albert Boime, The Academy and French Painting in the Nineteenth Century, New York, Phaidon, 1971 ; Pierre Vaisse, La troisième République et les peintres, Paris, Flammarion, 1995, p. 99 et suivantes.

12. On emprunte la notion de « nébuleuse réformatrice» à Christian Topalov (dir.), Laboratoires du nouveau siècle. La nébuleuse réformatrice et ses réseaux en France, 1880-1914, Paris, EHESS, 1999.

13. Cf. Debora L. Silverman, L'art nouveau en France. Politique, psychologie et style fin de siècle, Paris, Flammarion, 1994 (1989).

14. Sur la formation de cette distinction, cf. en particulier Nathalie Heinich, « De l'apparition de 1" 'artiste" à l'invention des ''beaux-arts"' ", Revue d'histoire moderne et contemporaine, vol. XXXVII, janvier-mars 1990. La remise en cause de cette forme de classification est très présente dans les projets artistiques révolutionnaires de l'abbé Grégoire qui, entre autres dans son rapport sur le conservatoire des arts et métiers, appelle à « censurer la division antique des arts en mécaniques et libéraux». Dans les années 1880, la référence des républicains à cette origine révolutionnaire est très fréquente.

15. La résolution du congrès de 1869 préconise ainsi « une éducation complète et générale en matière d'art, qui puisse en propager les notions les plus saines dans toutes les classes de la Société ». 
Après l'interruption de la guerre de 1870 , le mouvement reprend, notamment avec la formation en 1877 d'une Société pour la création d'un musée des arts décoratifs. C'est après une nouvelle exposition universelle, celle de $1878^{16}$, et dans une période marquée à la fois par la récession économique qui affecte particulièrement les industries d'art et la diversification des domaines dans lesquelles se jouent les concurrences internationales que la question des arts décoratifs et industriels est relancée, et intervient sur l'agenda politique ${ }^{17}$. Des revues sont créées, comme la Revue des arts décoratifs, en 1880. La même année, l'art décoratif est introduit dans le cours d'histoire de l'art de Charles Blanc au Collège de France. L'année suivante, paraît le premier des huit volumes du Dictionnaire encyclopédique et biographique de l'industrie et des arts industriels ${ }^{18}$. Le mouvement composite aux ramifications multiples rassemblant l'Union des beaux-arts appliqués à l'industrie, la Société du musée des arts décoratifs et leurs divers soutiens prend alors la forme d'une "nébuleuse réformatrice »: quand bien même ils n'occupent pas nécessairement des positions centrales dans le champ artistique, ses membres tentent, avec l'appui de parlementaires, de faire advenir de nouvelles définitions des problèmes artistiques préparant de nouvelles formes d'action publique.

Les membres de cette «coalition de ministres du gouvernement, d'aristocrates collectionneurs, de conservateurs de musée et d'un petit cercle de producteurs dans le secteur du luxe ${ }^{19}$ entendent revaloriser les « arts utiles» et ainsi réaffirmer l'intérêt socioéconomique de l'art. Des critiques d'art n'hésitent pas à « se placer sur le terrain même des économistes et à montrer par des chiffres que 1'art est un genre d'industrie supérieure $»^{20}$. Dans la presse généraliste, des articles plaident pour le développement des arts appliqués en montrant l'intérêt économique qui s'y attache ${ }^{21}$.

Cette vision économique de l'art, qui ne s'applique guère à la littérature ou à la musique, est bien faite pour séduire les agents du champ politique qui défendent, avant ceux de l'art, les " intérêts nationaux » et ceux de l'économie ou qui, pour mieux le servir, affirment comme Antonin Proust que «le culte des arts ne constitue pas seulement l'une des plus pures jouissances de l'homme, mais qu'il est encore l'un des plus puissants auxiliaires de la fortune des nations ${ }^{22}$. Les promoteurs de ces représentations se recrutent en particulier parmi les républicains opportunistes, dont certains - notamment autour de Gambetta - se définissent par leurs déplacements incessants dans l'espace social et leur capacité à allier la fréquentation des différentes élites à leur proximité au peuple, et sont par là même prédisposés à remettre en

16. Voir par exemple Jules Simon, Introduction au rapport du jury international. Exposition universelle de 1878, Paris, Imprimerie Nationale, 1880, cité in Stéphane Laurent, Les arts appliqués en France. Genèse d'un enseignement, Paris, éditions du CTHS, 1999.

17. La référence insistante à l'avance des pays étrangers et les voyages d'étude de spécialistes (comme Ferdinand Dutert ou Marius Vachon) pour le compte du gouvernement indiquent bien ce que la construction politique de ce problème doit à des logiques internationales.

18. Eugène-Oscar Lami, Dictionnaire encyclopédique et biographique de l'industrie et des arts industriels, Lami et Weiprecht, Paris, 1881-1888, 8 vol. Eugène Lami, officier d'Académie, etait attaché au service historique et des beaux-arts de la ville de Paris. Le dictionnaire rassemble des articles techniques, des biographies d'artistes, des monographies d'institutions.

19. Debora L. Silverman, L'art nouveau en France, op. cit., p. 118.

20. Eugène Véron, L'Art, Tome XVII, 1881, p. 114. Voir aussi Marius Vachon, « La situation actuelle des industries d'art en France », Gazette des beaux-arts, XXV, février 1882, p. 154-164.

21. C'est le cas du correspondant artistique de La République française Philippe Burty, qui rédige par ailleurs dans le premier volume du Dictionnaire encyclopédique et biographique de l'industrie et des arts industriels (op. cit., 1881) un article en forme de plaidoyer sur 1' " Art appliqué à l'industrie ». Le critique d'art du journal $L a$ France fait valoir que « les importations des produits d'art industriel en France se sont élevées en 1879 à la somme de 33 millions de francs tandis qu'en 1869 elles n'atteignaient que le chiffre de 11 millions ». Celui du Voltaire cite en exemple le cas anglais. Articles cités dans Le Temps, 2 novembre 1881, p. 2.

22. Antonin Proust, L'art sous la République, op. cit., p. 1. 
cause les frontières établies de l'art, qui plus que toute autre symbolisent la coupure entre les élites et le peuple ${ }^{23}$. Et l'on comprend que Gambetta ou Proust, qui fréquentent autant des industriels que des artistes, puissent être enclins, au travers de l'unité des arts, à promouvoir une vision économique de l'art et à intégrer des considérations esthétiques à la production industrielle et au commerce international. Leurs soutiens sociaux (les « couches nouvelles ») doivent précisément leur position au passage par les établissements d'enseignement spécialisé et les écoles d'arts et métiers qui sont au cœur du rapprochement de l'art et de l'industrie ${ }^{24}$

Socialement et politiquement fondée dans les trajectoires, les relations et les soutiens de Gambetta et de son entourage, l' « union des arts » forme en quelque sorte le prolongement esthétique d'une République définie comme l'union des classes sociales au sein de laquelle les entrepreneurs économiques — desquels sont rapprochés les artistes ${ }^{25}$ — jouent un rôle prépondérant. Une affinité relie de fait l'unité des arts à la vision du monde social de l'unanimisme et de l'économisme républicains qu'incarne Gambetta, où les frontières entre les objets et entre les hommes s'estompent derrière la recherche de la prospérité collective.

C'est très largement dans cette perspective que s'inscrit le développement de l'intervention publique en matière d'enseignement du dessin à partir de 1878. L'introduction de l'enseignement artistique obligatoire dans l'instruction secondaire, du dessin dans le certificat d'études primaires élémentaires, dans les écoles primaires supérieures, la loi sur les écoles professionnelles du 11 décembre 1880 sous le régime de laquelle sont placées, entre autres, l'école Boulle pour le mobilier et l'école Estienne pour les métiers du livre, forment de fait autant de mesures destinées à « intégrer l'art dans l'appareil socio-économique, relever l'art industriel et finalement vaincre la concurrence étrangère ${ }^{26}$. L'introduction des arts industriels dans l'enseignement artistique, défendue notamment par Philippe de Chennevières, le directeur de l'école nationale des beaux-arts, est débattue au sein du conseil supérieur des beaux-arts. Elle est considérée à la fois comme un moyen d'éviter la production d'artistes " déclassés » — c'est l'expression utilisée à l'époque - faute de trouver des débouchés pour leur production, et d'améliorer l'industrie, en lui offrant des ouvriers de qualité, d'éviter enfin que l'intervention artistique de l'État ne se réduise à «une assistance aux artistes nécessiteux ».

\begin{abstract}
«L'incubation artificielle et officielle d'artistes condamnés pour la plupart à ne pouvoir vivre de leurs œuvres est une des plaies de notre époque. Que d'hommes qui feraient dans l'industrie d'admirables ouvriers et qui ne sont, dans le domaine des beaux-arts, que des égarés, fatalement voués à réclamer les services d'une administration qui les a poussés dans une voie qui n'est pas la leur! Quelle perte pour nos industries, qui eussent pu trouver dans ces hommes de si utiles auxiliaires! Et, d'autre part, quel spectacle lamentable que celui d'une administration des beaux-arts réduite au rôle de bureau de bienfaisance ! » 27
\end{abstract}

Le soutien politique à la «cause » des liens entre l'art et l'industrie passe également par le relais que des parlementaires et des ministres offrent à la Société pour le musée des arts décoratifs. En 1879, des expositions au Louvre annoncent sa création prochaine, en vue de laquelle le sous-secrétaire d'État aux Beaux-Arts Édouard Turquet présente une proposition

23. Pour une présentation des « réseaux gambettistes », voir notamment Jérôme Grévy, La République des opportunistes (1870-1885), Paris, Perrin, 1998

24. Cf. Christophe Charle, Histoire sociale de la France au XIXe siècle, Paris, Seuil, 1991, p. 130.

25. La réforme du Salon évoquée plus haut ou le soutien à la création de sociétés d'artistes s'intègrent en effet à une conception de l'artiste comme entrepreneur économique indépendant. Cf. Patricia Mainardi, The End of the Salon, op. cit., p. 135.

26. Stéphane Laurent, Les arts appliqués en France, op. cit., p. 246.

27. Le Temps, 2 novembre 1881, p. 2. 
de crédit extraordinaire de cent cinquante mille francs l'année suivante. Début 1881, Antonin Proust, membre de la Société, dépose une proposition de loi pour la création d'un musée d'art industriel, arguant du retard pris par la France par rapport à ses concurrents étrangers Angleterre, Allemagne et Autriche notamment. La majorité parlementaire refuse d'engager les crédits nécessaires et retarde la création du musée - qui n'ouvrira ses portes qu'en 1905. Ce projet, tout comme le mouvement de réforme de l'enseignement du dessin, n'en contribue pas moins à structurer les débats et mobilisations pour l' " union des arts » qui donnent lieu à un ensemble si ce n'est coordonné, au moins convergent, de prises de position en faveur de la création d'un département ministériel dans le courant de l'année 1881.

\section{Des mobilisations pour un ministère}

Les projets de réforme du système d'action publique pour les arts, engagés dès 1870 et multipliés au cours des années suivantes, sont tout d'abord le fait de parlementaires et réformateurs d'État. Parmi eux, le rapport parlementaire d'Édouard Charton en 1875 conduit à un nouveau regroupement de services rattaché à l'Instruction publique. Celui de Lambert de Sainte-Croix, en 1878, suggère diverses mesures pour améliorer la cohésion de l'administration des beaux-arts: regroupement des services et répartition par fonction (conservation, enseignement, production), consolidation de la position du directeur des beaux-arts (auquel est réservée la présidence de tous les conseils et commissions), renforcement de l'inspection des beaux-arts, normalisation du recrutement des fonctionnaires. Jugés insuffisants ou seulement partiellement appliqués, ces deux rapports n'ont pas clos le débat d'une réforme qui reste à l'ordre du jour. Ils ont cependant produit des effets. Leur préparation a mobilisé des fonctionnaires et des parlementaires réunis en commission. À cette occasion, des informations ont été accumulées, des principes formalisés, des modes d'organisation repensés, offrant ainsi des points d'appui utiles à une entreprise institutionnelle plus ambitieuse

Celle-ci devient tout particulièrement d'actualité dès lors que s'est diffusée la croyance dans l'utilité de l'art pour l'économie nationale. La création d'un ministère des Beaux-Arts est ainsi présentée comme une nécessité par Antonin Proust dans son rapport du budget des beaux-arts en 1879: 1'exposition universelle de 1878 a démontré selon lui que «les autres peuples sont non plus des imitateurs mais des rivaux » pour les industries d'art françaises, le soutien coordonné de l'État étant dès lors présenté comme une nécessité pour lutter contre ces nouveaux concurrents. Antonin Proust reprend ces arguments dans le rapport du budget des beaux-arts de 1880, puis dans l'exposé des motifs d'un projet de loi sur l'organisation des écoles et musées d'art industriel, déposé au bureau de la Chambre en avril 1880. Ces préconisations parlementaires ne sont pas entièrement nouvelles, et d'autres partiellement similaires suivront mais, à la différence des autres, elles s'articulent directement à des prises de position analogues à l'extérieur du champ politique, notamment parmi les critiques d'art, les artistes et dans la presse.

La mobilisation est engagée par des critiques, recrutés notamment parmi les promoteurs des arts décoratifs et industriels et dans des revues d'art. Il s'agit en particulier des rédacteurs de la revue $L^{\prime} A r t$ qui, en 1876, avaient fait campagne pour l'établissement du musée des arts décoratifs et lancé une souscription en ce sens, rejoints dans leur entreprise par leurs 
homologues de la Gazette des beaux-arts et du Moniteur universe 28. L'appel à la création d'un ministère des Arts s'inscrit dans le prolongement de cette première mobilisation. $\mathrm{Au}$ début de l'année 1881, le critique d'art Paul Leroi dénonce l' " erreur funeste » que constitue selon lui le «démembrement » du ministère créé à la fin du second Empire : en plaçant les beaux-arts sous de multiples tutelles - Instruction publique mais aussi Travaux publics et Cultes - cette réorganisation aurait conduit à une paralysie préjudiciable à la conservation et à l'exposition des œuvres d'art ${ }^{29}$. L'article, qui conclut à l' « impossibilité de maintenir [cette] organisation hybride », est suivi par des appels plus directs à la création d'un " ministère des Arts $\gg{ }^{30}$. Le journal des arts s'associe à ces appels à l'automne $1881^{31}$ et, avec L'Art, adresse le 14 octobre une pétition signée par quatre-vingt dix-neuf artistes et critiques au président de la Chambre des députés, Léon Gambetta.

« Monsieur le Président, Il y a peu d'années, l'art était considéré en France comme une chose de simple agrément ou de pure vanité, en tout cas comme une affaire de luxe n'intéressant qu'un petit nombre d'amateurs. Mais depuis que les expositions universelles ont permis d'étudier et de comparer les forces productives des peuples, on s'est aperçu que l'art exerce une véritable fonction sociale par son indiscutable influence sur le chiffre des échanges internationaux, et par conséquent sur la richesse publique.» [Suit un argumentaire très économique].

Le texte de la pétition se termine en appelant à la «constitution d'un ministère des Arts auquel on rendrait toutes les parties qui ont été distraites, pour être données » à d'autres départements ${ }^{32}$

Ces critiques d'art et artistes favorables aux arts décoratifs parviennent à rallier à leur cause d'autres composantes du champ artistique. «Cette création était attendue, désirée, provoquée par presque tous les hommes qui se sont fait une spécialité de l'étude des questions d'art », estime après coup le critique et chroniqueur de la vie artistique Victor Champier ${ }^{33}$. Au-delà des revues d'art, ce projet est soutenu par la presse généraliste ${ }^{34}$. Les critiques d'art de la plupart des grands titres de la presse de l'époque sont en effet actifs dans le mouvement des arts industriels ${ }^{35}$. Plus largement, des associations ouvrières, des chefs de la grande industrie et des municipalités prennent publiquement position pour la création d'un ministère. Dans le

28. La revue L'Art est fondée en 1875 par le journaliste Eugène Véron. Né en 1825, agrégé de lettres, celui-ci écrit dans divers journaux de la presse républicaine nationale et locale (il a notamment été rédacteur en chef au Progrès de Lyon). Il est par ailleurs membre du Conseil supérieur des beaux-arts à partir de 1880 .

29. Paul Leroi, « Le sous-secrétariat des Beaux-Arts », L'Art, Tome III, 1881, p. 231-234.

30. Une série d'articles intitulée « Le ministère des Arts » paraît dans L'Art, Tome XVII, 1881, p. 49-56 (Paul Leroi), p. 89-93 ; 114-115 et 158-159 (Eugène Véron).

31. Le 21 octobre, l'éditorial du rédacteur en chef Auguste Dalligny est titré : «Un ministère des Beaux-Arts SVP ». Il reprend les arguments développés dans $L$ 'Art, plaide pour le développement des arts décoratifs et le rapprochement des arts et des sciences. Celui de la semaine suivante s'engage plus directement dans la préconisation de modes d'organisation pour le futur ministère.

32. Reproduit in Le journal des arts, 15 novembre 1881.

33. Victor Champier, L'année artistique, op. cit., p. 7. Le jeune critique d'art Victor Champier (il est né en 1851) est très présent dans les milieux des arts industriels. En 1878, il est secrétaire de la Société pour le musée des arts décoratifs ; secrétaire de rédaction de la revue 1'Art, il fonde la Revue des arts décoratifs en 1880 . Il deviendra plus tard (en 1902) directeur de l'école nationale des arts industriels de Roubaix.

34. « Un grand nombre de journaux ont bien voulu se joindre à nous dans la campagne que nous avons ouverte pour la création d'un ministère des Arts », se félicite le rédacteur en chef de L'Art. Eugène Véron, « Le ministère des Arts », art. cit., p. 114.

35. C'est le cas notamment de Philippe Burty, membre de 1'Union centrale des beaux-arts appliqués à l'industrie et auteur de deux ouvrages sur les arts industriels (La République), de Victor Champier (Le Moniteur universel), de Paul Mantz (Le Temps), et de Marius Vachon (La France), auteur de très nombreux écrits sur les arts industriels dont il deviendra l'un des principaux promoteurs dans les années 1910. 
rapport proposant cette création, Léon Gambetta peut ainsi faire référence à « un véritable mouvement d'opinion ${ }^{36}$. Les articles du Temps au cours du mois d'octobre 1881 donnent une indication de ces soutiens ${ }^{37}$. Un premier texte, en une de l'édition du 18 , présente de manière favorable mais mesurée la possibilité de créer un ministère des Beaux-Arts. Cette création «ne ferait pas seulement honneur à notre temps mais aurait une portée politique et sociale, parce qu'elle tendrait à transformer dans l'une de ses branches les plus importantes les conditions du travail national ». Trop timide, cet article suscite de nombreuses lettres de lecteurs et oblige le journaliste à se justifier et à renchérir trois jours plus tard :

« Nous ne partageons nullement les préventions que l'on paraît avoir dans les rangs du parti républicain contre l'accroissement du nombre des départements ministériels. Nous estimons, au contraire, que dans une démocratie comme la nôtre, on ne saurait trop multiplier les responsabilités. »

L'article évoque alors la possibilité d'un «ministre des arts et de l'industrie » de manière positive.

«Il faudrait de parti pris se refuser à l'évidence pour ne pas reconnaître qu'il est urgent d'élargir les bases de [1'administration des beaux-arts] et d'étendre ses attributions si nous ne voulons pas que nos grandes industries soient victimes d'une inaction déjà trop prolongée. En permettant à l'administration des beaux-arts remaniée de prendre sous sa tutelle les conservatoires, écoles d'art et musées d'art industriel, de faire appel au concours des départements, des communes, des chambres de commerce, des comités d'art et d'architecture et des syndicats professionnels, il n'est point douteux que l'on arrive promptement à un résultat qui transformera les conditions du travail national et rendra à nos industries artistiques la supériorité qu'elles ont eue pendant si longtemps. » ${ }^{38}$

36. Léon Gambetta, «Rapport au président de la République pour la création d'un ministère des Arts », Journal Officiel, 14 novembre 1881, reproduit en annexe. On ne peut prétendre dans le cadre de cet article établir avec toute la précision qui serait nécessaire les origines, les conditions et les modalités de ces soutiens. Sans doute les articles de presse et autres discours sur lesquels on s'appuie, eux-mêmes favorables à la création d'un ministère, en fournissent-ils une vision tout particulièrement unanimiste. L'insistance avec laquelle des sources diverses font état de l'ampleur et de la diversité de prises de positions favorables fournit toutefois un indice de ce qu'elles ne se cantonnent pas à la fraction mobilisée la plus visible.

37. Ces articles, non signés, sont très certainement rédigés par Paul Mantz, qui sera nommé directeur de la Conservation lors de la création du ministère. Sur Paul Mantz, Cf. infra.

38. Le Temps, 21 octobre 1881. 


\section{Les conditions politiques d'un projet artistique} «a question, mûre depuis une éternité pour tout esprit clairvoyant et soucieux de véritables intérêts du pays,
l'est enfin devenue pour les plus aveugles. [... J Jamais l'occasion n'a été opportune comme elle l'est
aujourd'hui à la veille de la nomination d'un nouveau ministère. »39

Les projets de réforme administrative de l'action publique pour les arts et les mobilisations initiées au sein de la «nébuleuse réformatrice» des arts industriels se rejoignent et s'affermissent dans la conjoncture politique particulière de la fin 1881. Le « grand ministère » de Léon Gambetta permet en effet la réalisation de nouvelles manières de penser et d'organiser l'action de l'État. Dans cette ouverture des possibles, les ressources artistiques et politiques personnelles d'Antonin Proust prennent toute leur valeur, et peuvent être investies dans la fondation d'une politique artistique gouvernementale.

\section{Le " grand ministère » de Gambetta et l'ouverture du champ des possibles}

Le « grand ministère » de Gambetta constitue une configuration gouvernementale exceptionnelle qui s'inscrit dans une période particulièrement chargée et tendue politiquement ${ }^{40}$. Les grandes lois et projets de réforme des années 1880-8141 s'accompagnent de vifs affrontements parmi les républicains. Aux oppositions entre les opportunistes et les radicaux-socialistes réunis autour de Clemenceau s'ajoutent ainsi celles qui se jouent au sein du camp opportuniste, entre la gauche républicaine réunie autour de Jules Ferry — président du Conseil depuis septembre 1880 - et l'Union Républicaine de Léon Gambetta - qui préside la chambre des députés depuis janvier 1879 .

Plusieurs fois écarté de la présidence du conseil par le président de la République Jules Grévy, Léon Gambetta occupe une position définie à l'époque comme celle d'un "président du conseil occulte », entouré de journalistes, d'artistes, de hauts fonctionnaires et de représentants des milieux d'affaires, présenté comme le partisan d'une dictature par ses adversaires. Après les législatives du mois d'août, il est finalement pressenti pour former un gouvernement. Au cours de la période de latence qui sépare ces élections de la rentrée parlementaire, fin octobre, Gambetta commence à élaborer un projet de ministère avec les principaux représentants républicains ${ }^{42}$. Le ralliement de l'aile gauche des opportunistes à Clemenceau, puis la cristallisation des oppositions autour du débat sur la Tunisie qui, fin octobre, précipite la démission du gouvernement Ferry, remettent en cause cette coalition. Dès lors, Gambetta ne peut plus recruter les membres de son cabinet que parmi ses proches, souvent sans position politique importante. «Les principaux chevaux de l'attelage rentrent tous au hangar, écrit-il, et je me vois obligé aujourd'hui de recommencer le casse-tête chinois

39. Paul Leroi, « Le ministère des Arts », art. cit., p. 54.

40. À ce propos voir notamment Maurice Agulhon, La République, I. 1880-1932, Paris, Hachette coll. Pluriel, 1990, p. 60-63, et Jérôme Grévy, La République des opportunistes, op. cit.

41. Gratuité de l'enseignement primaire, liberté de la presse, légalisation des syndicats ouvriers, abaissement du service militaire à trois ans, réforme de la magistrature, institution d'un impôt général sur le revenu, création d'une caisse d'assurance en faveur des invalides du travail, etc.

42. Freycinet à la Guerre, Ferry à l'Instruction publique, Léon Say, président du Sénat, aux Finances. Brisson, Rouvier, Challemel-Lacour entre autres sont également pressentis. La formation d'un tel gouvernement est alors débattue depuis déjà plusieurs mois. D'après Joseph Reinach, l'idée d'un « grand ministère » aurait été inventée dans une intention polémique par les journalistes du Figaro, et rétrospectivement imputée à Gambetta. Joseph Reinach, Le ministère Gambetta, histoire et doctrine (14 novembre 1881-26 janvier 1882), Paris, Charpentier, 1884, p. 61-62. Sur les longues tractations en vue de la formation de ce gouvernement, voir p. 67 et suivantes. 
avec des mandarins de second rang. » Mais «au moins ceux-là seront disciplinés et homogènes ${ }^{43}$. L'appellation " grand ministère » s'emploie désormais par dérision, pour désigner ce « ministère de commis $»^{44}$

Comprenant douze ministres et neuf sous-secrétaires d'État, le gouvernement est plus étoffé que ceux de l'époque, qui comprennent en général dix ministres et quelques secrétaires d'État ${ }^{45}$. Avec un âge moyen de quarante-neuf ans - Gambetta a quarante-trois ans - le gouvernement est sensiblement rajeuni par rapport à ceux qui le précèdent et le suivent immédiatement. Ses partisans le présentent comme un ministère de « spécialistes, dûment préparés par leurs études antérieures au portefeuille qui leur était confié ${ }^{46}$ ». Léon Gambetta se charge des Affaires étrangères, avec Spuller comme sous-secrétaire d'État. WaldeckRousseau, alors jeune député (il a trente-cinq ans), avocat, est nommé à l'Intérieur. Des proches sont nommés aux Finances (Allain-Targé) aux Travaux Publics (Raynal) et au Commerce (Rouvier). Des officiers également proches de Gambetta sont affectés à la Guerre (le général Campenon) et à la Marine (le capitaine de vaisseau Gougeard). Le sénateur Cazot est nommé à la Justice, Paul Bert, à l'Instruction publique.

Ce recrutement particulier et la mise entre parenthèses du jeu parlementaire dans lequel s'opère habituellement la formation d'un gouvernement procurent une marge de manœuvre inédite qui facilite la redistribution des compétences gouvernementales. Outre l'augmentation du nombre des postes, plusieurs innovations institutionnelles interviennent. Alors qu'elles ne constituaient qu'une direction au ministère de la Marine, les Colonies se voient dotées d'un sous-secrétariat confié à Félix Faure, alors jeune industriel nouveau venu en politique ${ }^{47}$. Deux départements ministériels sont créés: l'Agriculture, désormais séparée du Commerce et confiée à Devès, et le ministère des Arts attribué à Antonin Proust. Facilitée par la conjoncture politique, cette redistribution s'inscrit plus largement dans un vaste projet de réformes dont Gambetta a fait son programme. Entre autres, la nouvelle division du travail gouvernemental doit ainsi réduire le sureffectif d'une «bureaucratie oiseuse », renforcer « l'autorité de l'État» et assurer la « responsabilité de l'exécutif», en appliquant «à chaque fonction une compétence qui soit à la hauteur de cette fonction ${ }^{48}$.

\section{Les ressources multiples d'Antonin Proust et l'invention d'une fonction ministérielle}

En ce qui concerne le nouveau ministre des Arts « M. Antonin Proust semblait se préparer depuis plusieurs années à ces hautes et délicates fonctions », estime Victor Champier ${ }^{49}$. Et de

43. Léon Gambetta, Lettres à Léonie Léon, successivement 12 et 14 novembre 1881.

44. «M. Gambetta a choisi tout simplement, dans son entourage, des gens de bonne volonté et de soumission ». (La Revue des deux mondes, cité in Jean Chastenet, Gambetta, op. cit., p. 342.) Il « s'est retourné pour faire appel à des personnalités moins marquées et moins marquantes, les plus aptes par cela même à recevoir son impulsion et à s'approprier ses vues; le ministère est moins grand, mais il y occupe une plus grande place ». Le Temps, 15 novembre 1881 , p. 1.

45. Cf. Pierre Rosanvallon, L'État en France de 1789 à nos jours, Paris, Seuil, 1993, p. 294.

46. Joseph Reinach, Le ministère Gambetta, op. cit., p. 84.

47. Sur le sous-secrétariat aux Colonies, cf. Raoul Girardet, L'idée coloniale en France, Paris, Hachette, 1978, p. 120 .

48. Léon Gambetta, discours à la Chambre des députés, 8 décembre 1881. Pour une présentation — partisane des conceptions de l'organisation et de la fonction gouvernementales défendues par Gambetta, voir Joseph Reinach, Le ministère Gambetta, op. cit., p. 91 et suivantes. Sur les réformes mises en œuvre au moment du ministère Gambetta, cf. ibid., p. 170 et suivantes (183-190 pour ce qui concerne plus précisément le ministère des Arts).

49. Victor Champier, L'année artistique, op. cit., p. 10 
fait, Antonin Proust disposait indéniablement de ressources ajustées à ce poste: des ressources politiques, liées notamment à sa proximité avec Gambetta ; un capital relationnel et un crédit symbolique obtenus dans le champ artistique comme critique et « ami des arts ». Ces deux séries de ressources ont de plus été largement investies — et par là même développées - dans de multiples positions et entreprises liées au traitement public des questions artistiques.

Né à Niort en 1832, Antonin Proust est issu d'une famille de l'élite politico-administrative : son grand père était receveur général des Deux-Sèvres, son père, polytechnicien, a été député libéral sous la monarchie de juillet. Sa formation et ses activités sont typiques du personnel politique républicain des débuts de la IIIe République : après des études au lycée Henri IV et à la faculté de Droit de Paris, il devient avocat et publiciste. Il publie entre autres des travaux sur la Révolution à Niort et un recueil de lettres de Bismarck. Correspondant du Temps pendant la guerre de 1870, il est chargé de la politique étrangère dans la presse gambettiste La République Française et La Petite République ${ }^{50}$. Après avoir été le secrétaire particulier de Gambetta, il est élu en 1870 au conseil municipal de Niort. Il devient ensuite maire de cette ville, puis député des Deux-Sèvres en 1876. À la Chambre, il intervient fréquemment à propos $d$ 'art et de politique étrangère.

Parallèlement, Antonin Proust poursuit diverses activités artistiques. Il débute des études de peinture en 1850, dans les ateliers de deux «maîtres » de l'époque, les peintres Henri Decaisse et Thomas Couture. Dans les années 1860, il publie textes et dessins dans Le Tour $d u$ Monde, à propos de son voyage à Athènes. Dans L'Illustration, il fait paraître des dessins sur la guerre de 1870. Il écrit ensuite des récits, croquis et tableaux de mœurs sous le pseudonyme d'Antonin Barthélemy ${ }^{51}$. Collectionneur d'art, c'est aussi un critique connu pour ses monographies ${ }^{52}$ et surtout pour sa défense de l'impressionnisme dans les comptes-rendus des Salons qu'il publie dans les années 1870-80. Il entretient des relations étroites avec des artistes très différents : Édouard Manet, qu'il a rencontré au cours de ses études de peinture et qui fera trois portraits de lui — dont deux exposés au Salon, en 1880 et 1882 — Pierre Puvis de Chavannes, Auguste Rodin - qui sculpte son buste — ou Émile Gallé — qui réalise un gobelet en son honneur pour l'exposition de l'Union centrale des arts décoratifs en 1884. Il est enfin comme on l'a vu très actif dans le milieu des arts décoratifs et industriels.

Doté d'importantes relations et compétences artistiques, la légitimité d'Antonin Proust à occuper la fonction nouvelle de ministre des Arts est largement reconnue dans la presse et la critique d'art.

« C'est un lettré qui surveille nos théâtres, et avant de juger les artistes il a tenu la palette et manié le pinceau. " ${ }^{53} \mathrm{C}$ 'est « un écrivain de talent et un homme de goût depuis longtemps familiarisé avec les choses de son nouveau domaine " ${ }^{54}$. "Très versé dans les questions d'art, plein d'activité et de dévouement pour la cause des progrès et pour les intérêts particuliers des artistes, M. Antonin Proust est généralement considéré, pour ses aptitudes

50. Mémorial des Deux-Sèvres, 25 mars 1905, reproduit in Discours et articles de journaux prononcés et publiés à l'occasion des obsèques de M. Antonin Proust, Niort, 1905, $24 \mathrm{p}$.

51. Voir par exemple Un philosophe en voyage, par Antonin Barthélemy : Londres et les Anglais, en Grèce, les Moines byzantins, Contes orientaux : l'Immortalité, le Dracophage, Paris, Charpentier, 1864.

52. Voir par exemple Les beaux-arts à l'exposition de Londres, La Rochelle, impr. de A. Siret, 1862 ; Les beauxarts en province, Niort, impr. de Th. Mercier, 1867. De nombreuses autres paraîtront après son passage au gouvernement.

53. Jules Claretie, Le Temps, 27 décembre 1881.

54. Journal des arts, 18 novembre 1881 
personnelles, comme un des hommes les plus capables de donner satisfaction au monde artistique. ${ }^{55}$

Ce crédit dans les milieux artistiques est d'autant plus fort que cet « artiste et esthète de la jeune République ${ }^{56}$ a multiplié les investissements institutionnels et politiques dans les questions artistiques. Réciproquement, ces investissements lui confèrent la position d'un spécialiste reconnu des questions artistiques au sein du champ politique. Dès 1870 , il participe à l'inventaire des richesses artistiques de la France dirigé par Philippe de Chennevières, et multiplie ainsi les relations avec des amateurs, collectionneurs d'art et érudits. Député, il devient membre de la commission des monuments historiques (dont il est vice-président en 1879), de la commission des théâtres, du conseil supérieur des beaux-arts, et de la commission chargée de préparer la réorganisation des services administratifs des beaux-arts (qui donne lieu au rapport Sainte-Croix en 1878). Régulièrement rapporteur du budget des beaux-arts (en 1879,1880 puis en $1884,1885,1886,1887,1891$ et 1892), il rapporte ou présente également des projets de loi dans le domaine artistique (notamment sur les écoles et musées d'art industriel).

Cette reconstitution de l'économie des ressources d'Antonin Proust ne saurait permettre à elle seule de saisir pleinement les conditions de sa nomination au poste de ministre des Arts. Il faut en premier lieu rappeler que le rapport entre un nouveau poste ministériel et son titulaire est dialectique : celui de ministre des Arts a été fait pour et par Proust tout autant que ce dernier était préparé à l'occuper ${ }^{57}$. Ensuite, les ressources de Proust prennent place dans l'espace des concurrences entre prétendants possibles. S'il était sans doute l'un des plus investis dans le traitement des questions artistiques, il n'était pas pour autant le seul agent du champ politique à pouvoir alors se prévaloir des compétences et relations susceptibles de le rendre "ministrable » ${ }^{58}$. Les prévisions-prescriptions des critiques d'art mobilisés pour la création du ministère en témoignent, qui évoquent le sous-secrétaire d'État aux Beaux-Arts Edmond Turquet ${ }^{59}$ ou le député Édouard Lockroy, rapporteur du budget des beaux-arts pour 1881 et $1882^{60}$.

Ce sont les événements politiques qui ont, en un temps très court, restreint l'espace des prétendants possibles aux proches collaborateurs de Gambetta. C'est dire que la valeur des ressources de Proust se constitue pour une part décisive dans la conjoncture particulière que marque la coïncidence entre des mobilisations pour une politique artistique et la configuration politique et institutionnelle du "grand ministère ${ }^{61}$. C'est dans cette combinaison entre un état des rapports entre champs artistique et politique favorable, un jeu politique qui ouvre des

55. L'Artiste, 1881, p. 574.

56. Gabriel Hanotaux, Mon temps, t. II, Paris, Plon, 1938, p. 127-128.

57. Ce rapport d'ajustement mutuel entre le poste et les dispositions de son (ses) titulaire(s) n'est bien sûr pas spécifique aux fonctions gouvernementales et s'observe dans bien d'autres cas d'invention de fonctions ou professions nouvelles. Cf. par exemple l'analyse en ce sens de Francine Muel-Dreyfus, Le métier d'éducateur. Les instituteurs de 1900, les éducateurs spécialisés de 1968, Paris, Minuit, 1983.

58. Voir par exemple Jean Estèbe, Les ministres de la République, 1871-1914, Paris, Presses FNSP, 1982 ; pour la période ultérieure, François Grèze-Rueff, La culture des députés français (1910-1958), Toulouse, Presses universitaires du Mirail, 1994

59. Paul Leroi, « Le ministère des Arts », art. cit.

60. L'Artiste, 1881. Édouard Lockroy deviendra plus tard ministre du Commerce et de l'industrie, et ministre de l'Instruction publique et des beaux-arts.

61. Pour importantes qu'elles aient pu être, ces ressources n'ont du reste que peu été mises à profit par Antonin Proust en dehors de ces quelques mois. S'il a continué à être actif et à occuper des positions importantes dans le milieu des arts décoratifs, il n'a de fait jamais assumé de nouvelles responsabilités gouvernementales, ni même plus généralement dans le champ politique. 
possibles et la position devenue stratégique d'Antonin Proust que se forme le ministère des Arts. Et c'est dans cette combinaison que les ressources et dispositions particulières de Proust contribuent directement à la définition de ce nouveau rôle gouvernemental.

\title{
L'objectivation institutionnelle d'une politique artistique
}

\author{
«La République, c'est la forme qui entraîne le fond. »62
}

La substitution d'un ministère des Arts au sous-secrétariat d'État aux Beaux-Arts marque la promotion des questions artistiques au rang d'objets à part entière de l'intervention publique. Mais la création de ce ministère est plus qu'une simple élévation des beaux-arts dans la hiérarchie politico-administrative des dossiers et des secteurs. De par sa forme même, organisée non plus par disciplines et domaines artistiques plus ou moins clairement établis mais autour d'un triptyque de fonctions - enseignement, conservation, décoration -, elle désigne un nouveau rôle artistique pour l'État républicain en formation. Cette invention de nouvelles fonctions étatiques passe par des éléments matériels, des locaux, une organisation interne, un personnel partiellement nouveau. En matière artistique comme ailleurs, cette " forme " que constitue l'organisation de l'institution gouvernementale doit " entraîner le fond », comme le dit Gambetta. En même temps qu'une nouvelle conception du rôle artistique de l'État, cette structure ministérielle inédite est en effet censée réaliser, au sens fort du terme, une nouvelle manière d'appréhender l'art, en refondant son "unité » et en respectant sa " liberté », ces deux principes sur lesquels le ministère est construit et que, par les vertus propres de cette construction, il doit faire advenir.

\section{L'invention de nouvelles fonctions étatiques}

Quand bien même ils ne sont pas tous directement tendus vers cet objectif, les projets et mobilisations antérieurs ont formé une base utile à la création du nouveau ministère. C'est le cas en particulier du rapport Lambert de Sainte-Croix: Antonin Proust a participé à sa préparation, et le texte de Gambetta pour la création du ministère y fait explicitement référence. De même, l'organisation du ministère reprend certaines préconisations - à commencer par sa dénomination — des critiques d'art, journalistes et artistes mobilisés pour sa création ${ }^{63}$. Le projet n'est donc pas radicalement inédit. Faute de temps, ou du fait des résistances qu'ils suscitent, tous les changements qu'il implique ne sont pas pleinement réalisés. Sa promotion en programme d'action gouvernementale et sa traduction, même partielle, en actes n'en marquent pas moins une innovation importante.

Avec la création du ministère, tout un ensemble d'aménagements et dispositifs bureaucratiques vient matérialiser la nouvelle politique gouvernementale. Ce sont en premier lieu des locaux. Il est prévu que le ministre s'installe dans les anciennes écuries impériales du

62. Léon Gambetta, discours au théâtre du Château d'eau, Paris, 20 octobre 1878, reproduit in Pierre Barral, Les fondateurs de la IIIe République, Paris, Armand Colin, 1968, p. 166-167. Voir aussi Claude Nicolet, La République en France, état des lieux, Paris, Seuil, 1992, p. 44.

63. Auguste Dalligny retrouve ainsi ses arguments et surtout ceux de la pétition dans le rapport adressé au Président de la République, la création du ministère des Arts provoquant selon lui « une pleine et entière satisfaction », Journal des arts, 18 novembre 1881. 
Louvre (ce qui ne se fait pas faute de crédits suffisants pour la réfection) et que les services nouvellement rattachés au ministère prennent place aux côtés des bureaux de l'administration des beaux-arts de la rue de Valois ${ }^{64}$. Un secrétariat général est mis en place, qui doit assurer la coordination des trois nouvelles directions. Enfin, un personnel d'encadrement administratif conforme aux orientations de la politique nouvelle est mis en place. Outre des fonctionnaires au profil habituel - $M$. Poulin, ancien chef de division des bâtiments civils au ministère des Travaux publics, et Adolphe Tétreau, maître de requêtes au conseil d'État et membre du Conseil supérieur des beaux-arts depuis 1876, sont respectivement nommés directeur de la Construction et secrétaire général ${ }^{65}$ — des spécialistes des questions artistiques remplacent les anciens administrateurs des beaux-arts suspects d'amitiés académiques. Le poste de directeur de l'Enseignement est ainsi confié à un jeune architecte, Ferdinand Dutert. Né en 1845, prix de Rome en 1869, ce dernier est déjà intégré à l'administration gouvernementale — il est inspecteur principal de l'enseignement du dessin depuis 1879.

Ce « jeune architecte d'un grand avenir [...] s'est particulièrement occupé des questions d'art industriel. Il a fait récemment un voyage en Angleterre qui a motivé des rapports du plus haut intérêt. M. Dutert n'accepterait, ajoute-t-on, des fonctions administratives que provisoirement, pour organiser le service de l'enseignement. Il aurait l'intention de reprendre ses travaux d'architecture après une année » (Le Temps).

Le nouveau directeur de la Conservation, Paul Mantz, est sans doute celui qui incarne le mieux la politique ministérielle. Né en 1821 , il a déjà derrière lui une double carrière administrative et artistique. Après des études de droit, il entre dans l'administration et, en janvier 1880, devient sous-directeur de l'administration départementale et communale au ministère de l'Intérieur. Par ailleurs, il publie à partir de 1844 des critiques d'art dans diverses revues et dans Le Temps, ainsi que plusieurs études historiques sur des thèmes liés aux arts industriels - notamment dans la Gazette des beaux-arts et le Dictionnaire des arts industriels. En 1877, il fait partie des membres fondateurs de la Société pour le musée des arts décoratifs. Ce parcours lui permet d'être salué lors de sa nomination comme un « écrivain et critique d'art éminent, bien connu [pour ses] idées avancées [...] que sa compétence exceptionnelle aurait dû faire enlever depuis longtemps au ministère de l'intérieur » (Journal des Arts, p. 12) ${ }^{66}$. Lors de sa nomination au ministère, il compte de fait parmi les principaux critiques d'art de l'époque.

La rationalisation de l'organisation administrative des beaux-arts et l'appel à des compétences spécifiques doivent contribuer à fixer les fonctions jusqu'alors fluctuantes des pouvoirs publics en matière artistique. Plus encore, celles-ci sont désormais établies dans des nomenclatures administratives et des formes institutionnelles. À la manière des listes étudiées par Jack Goody, qui permettent d'opérer de nouveaux rapprochements sur la base de catégories abstraites de l'expérience immédiate ${ }^{67}$, cette redistribution des compétences administratives a partie liée avec l'élaboration d'une " raison », en l'occurrence une " pensée artistique d'État ». Celle-ci s'ordonne autour de trois fonctions, assurées par trois directions, qui intègrent un ensemble d'objets et d'institutions au sein de la politique artistique gouvernementale. Chacun d'entre eux s'y voit ainsi assigner une place, non pas d'après la sectorisation des activités artistiques (arts plastiques, théâtre, etc.) mais selon la fonction que

64. Journal des arts, 18 novembre 1881 ; Le Temps, ler décembre 1881.

65. «L'organisation centrale du ministère des Arts », Journal des arts, 25 novembre 1881; Le Temps, 23 novembre 1881, p. 2.

66. Après le démantèlement du ministère des Arts, il est nommé directeur des beaux-arts, poste dont il démissionnera le 20 novembre 1882.

67. Jack Goody, La Raison graphique. La domestication de la pensée sauvage, Paris, Minuit, 1978. 
le gouvernement revendique à son endroit et-ou celle qu'elle lui assigne. La direction de l'Enseignement comprend ainsi, en plus des établissements d'enseignement artistique, ceux de l'enseignement technique, et les théâtres. Celle de la Conservation regroupe les musées, le mobilier national, la bibliothèque nationale, les monuments historiques, les cathédrales, les édifices diocésains, les archives et les expositions. Celle de la Construction et de la Décoration rassemble enfin les bâtiments civils, les palais nationaux, l'acquisition et les commandes d'œuvres d'art, la décoration des lieux publics et l'organisation des fêtes publiques.

$\mathrm{Au}$ moins dans un premier temps, les commentateurs qui prennent position sur ces changements s'accordent à le trouver positif. Le ministère a été

\begin{abstract}
« constitué sur de larges bases, d'après un plan méthodique, discutable peut-être mais à coup sûr bien étudié, et [a] centralisé dans une seule main les forces éparses, stériles parce qu'elles agissent le plus souvent en sens contraires, des diverses administrations relevant des beaux-arts par quelque côté» 68 . "La création d'un ministère spécial des Arts, embrassant résolument tout ce qui, de près ou de loin, se rattache à cet important service, vient de modifier avec bonheur, nous l'espérons, les bases fondamentales et de son action et de son organisation. Ses proportions administratives se trouvent singulièrement élargies et transformées, et ce qui se passait dans les cadres étroits d'avant-hier pourra à peine se reconnaître après-demain. ${ }^{69}$
\end{abstract}

Regroupées par fonction dans les nouvelles catégories de la vision étatique, les questions artistiques se trouvent désormais intégrées à des problèmes sociaux et économiques desquels elles étaient jusqu'alors traditionnellement « désencastrées » ${ }^{70}$. Présenté comme le « ministère du travail national », le ministère des Arts est de fait constitué autour de problèmes et principes tant économiques et sociaux qu'artistiques, entre lesquels il est précisément supposé établir des liens indissociables ${ }^{71}$. Ces liens sont assurés par la réunion des arts préconisée par les promoteurs des arts décoratifs et industriels, et que le ministère doit favoriser.

\title{
Réaliser " l'unité des arts »
}

Ministère des Arts et non pas des Beaux-Arts : la dénomination de la nouvelle institution ${ }^{72}$ dit bien la révolution symbolique dont, sur la base des mobilisations et transformations engagées par les promoteurs des arts industriels, elle est censée favoriser l'avènement. Cette révolution symbolique consiste en une rupture avec toute une série de représentations traditionnellement attachées à l'art: inutilité, luxe, élitisme, coupure d'avec l'artisanat et l'industrie. Son principe central, l'unité des arts, est sans cesse répété par Proust, dans le droit fil des conceptions des arts décoratifs et industriels :

68. Victor Champier, L'année artistique, op. cit., p. 3.

69. Charles-Philippe de Chennevières, Souvenirs d'un directeur des beaux-arts, première partie, Paris, Aux bureaux de l'Artiste, 1883, p. 1. Ce texte écrit en novembre 1881 a été initialement publié dans L'Artiste. L'ancien directeur des beaux-arts et de l'école des beaux-arts commence ses souvenirs par cette phrase ; c'est dire l'importance que pouvait revêtir la création du ministère au moins pour les agents les plus directement concernés.

70. On emprunte l'idée de désencastrement à Karl Polanyi, La grande transformation, Paris, Gallimard, 1984.

71. L'expression «ministère du Travail» est fréquemment employée pour désigner la fonction que ses promoteurs entendent assigner au ministère des Arts (comme l'amélioration des compétences professionnelles des ouvriers). Sur l'institution créée plus tard sous cette appellation, voir Jacques-André Tournerie, Le ministère du Travail : origines et premiers développements, Paris, Cujas, 1971.

72. Celle-là même qu'avaient préconisée Paul Leroi et Eugène Véron dans leurs articles. « Le ministère des Arts », art. cit. 
«Si l'art est un dans son inspiration, il est varié à l'infini dans ses manifestations, et [...] nous devons tenir en égale estime, depuis le chef d'œuvre qui traduit les plus nobles pensées jusqu'à l'instrument usuel qui nous séduit par sa forme, sa couleur et son dessin. ${ }^{73}$

Pour rétablir cette unité, Antonin Proust et ceux qui le soutiennent croient en l'effet performatif d'un changement dans les formes officielles de catégorisation : en inscrivant la vision réunifiée des arts dans les structures gouvernementales, ce sont selon eux les structures mentales - les représentations de l'art et de ses fonctions - et les structures sociales l'organisation de l'activité artistique et la diffusion de ses produits - que l'on pourra transformer. C'est l'un des arguments de Leroi à l'appui de sa campagne pour la création du ministère :

« Les beaux-arts ont de tout temps été - fort à tort, je le répéterai toujours - considérés comme affaire de luxe et plaisirs de désœuvrés. Créez le Ministère des Arts, établissez nettement son programme et vous aurez immédiatement raison de cette ridicule appréciation, chère aux seuls moutons de Panurge ${ }^{74}$.

Antonin Proust rappelle cette ambition dans le livre qu'il publie après son ministère.

« Les hommes qui arrivèrent au pouvoir, le 14 novembre 1881, étaient pénétrés de cette idée que l'art est un et qu'il ne convient de différencier les artistes que par le degré d'inspiration. Nous sommes victimes d'une mauvaise locution et d'une locution qui n'est pas d'ancienne date. C'est la haine des maitrises, c'est le dédain que celui qui se qualifiait d'artiste professait pour l'artisan, qui a donné naissance il n'y a pas deux cents ans, à cette distinction de l'art et des beaux-arts. [...] Mais notre siècle a accentué la division. Et c'est à notre siècle qu'il apparient de la faire disparaître et de confondre dans une même famille tous ceux qui servent l'art avec un égal degré de conviction. C'est dans cet intérêt d'union que fut constitué le ministère des Arts. ${ }^{75}$

La modification des structures administratives évoquée plus haut objective ainsi, en plus d'une fonction artistique gouvernementale, la (ré)vision des activités artistiques promue par le mouvement des arts décoratifs et les républicains réunis autour de Gambetta. Le ministère des Arts réunit de fait non seulement tous les services du sous-secrétariat d'État aux Beaux-Arts (école des beaux-arts, musées, expositions, manufactures, commandes et acquisitions, monuments historiques, théâtre), mais aussi les Bâtiments civils — qui proviennent du ministère des Travaux publics - et les édifices diocésains - jusqu'alors au ministère de l'Instruction et des Cultes. Plus encore, y sont intégrés des services considérés comme relevant des " arts mécaniques » et habituellement exclus de toute considération artistique. Il en va ainsi de l'enseignement du dessin dans les établissements scolaires qui relevait de l'Instruction publique et de l'enseignement technique des arts et métiers, détaché du Commerce et de l'agriculture.

En plus des structures ministérielles, l'unité des arts doit être favorisée par les nouvelles pratiques d'intervention qu'elles permettent. La modification de l'organisation des musées conduit ainsi à élargir la liste des musées nationaux, jusqu'alors limitée au Louvre, au Luxembourg, à Versailles et Saint-Germain, à des musées d'arts « mineurs » ou « utiles »-

73. Antonin Proust, Édouard Manet, souvenirs publiés par A. Barthélémy, Paris, Librairie Renouard, H. Laurens éditeur, 1913, p. 129.

74. Paul Leroi, « Le ministère des Arts », L'Art, Tome XVII, p. 54.

75. Antonin Proust, L'Art sous la République, op. cit., p. 11-12. 
les musées de sculpture comparée du Trocadéro, de céramique de Sèvres, des tapisseries des Gobelins, du conservatoire des arts et métiers - en plus d'autres plus classiquement " artistiques » - ceux de l'école des beaux-arts et de Cluny, du conservatoire de musique et de l'opéra $^{76}$. Une orientation similaire préside à des projets de réforme de l'enseignement artistique. Il est question d'instaurer un système d'enseignement regroupant trois catégories d'établissements : les écoles d'enseignement supérieur, dites «des beaux-arts »; les écoles d'enseignement secondaire, dites " des arts et métiers », "d'arts» ou « de profession »; les écoles de dessin et de modelage et cours spéciaux d'enseignement des principes généraux du dessin. En décembre 1881, il est décidé que les crédits destinés aux écoles municipales des beaux-arts seront utilisés pour transformer ces établissements en écoles d'arts appliqués ${ }^{77}$. Le ministère des Arts intervient encore pour développer et ouvrir aux arts décoratifs l'enseignement des arts dans les écoles primaires et dans les universités. Un vaste projet de réforme ouvrant l'enseignement à tous les arts, incluant l'architecture, restera sans lendemain. Enfin, pour favoriser le développement des arts industriels, une exposition internationale doit être organisée à Paris, du ler août au 15 novembre $1882^{78}$. Une commission d'enquête sur la situation des ouvriers et des industries d'art est instituée dès la fin 1881 , qui réunit des parlementaires, des conseillers municipaux parisiens, des responsables d'institutions (manufactures, écoles d'art, conservatoire des arts et métiers), auxquels s'ajoutent le directeur de Christophle, le vice-président de l'Union centrale des arts décoratifs et trois publicistes, dont Marius Vachon, militant et théoricien des arts décoratifs 79

\section{Garantir « la liberté de l'art»}

Porté par les promoteurs des arts décoratifs, le nouveau ministère se fonde sur le principe d'unité des arts; soutenu également par les fractions anti-académiques du champ artistique (les « artistes libres », comme les impressionnistes), c'est dans le même temps la liberté de création qu'il doit respecter et assurer. Antonin Proust forme ainsi un néologisme pour désigner le dessein de son ministère : il s'agit «d'indépendantiser l'art, surtout dans ses sphères supérieures ${ }^{80}$. Respectueuse de l'autonomie et de la diversité des artistes, la politique artistique gouvernmentale doit également en être garante. L'État, explique plus tard Antonin Proust, doit " se montrer impartial et ne point proscrire un artiste parce qu'il ne s'accorde pas de sentiment avec l'opinion dominante $» 81$.

Tout comme celle de l'unité de l'art, l'affirmation politique du principe de sa liberté est une manière de marquer la rupture avec le « despotisme monarchique » - l'expression « liberté de l'art » est en effet d'origine révolutionnaire. Le maintien de l'État dans un rôle de simple arbitre sans exclusive esthétique assuré par le ministère des Arts établissait « un régime de

76. Cette réorganisation fait suite au rapport sur les musées en Europe remis par l'historien d'art Olivier Rayet en 1881. Prévue par le décret du Président de la République sur proposition d'Antonin Proust du 24 janvier 1882 (Journal Officiel du 26 janvier 1882), le gouvernement étant renversé, elle n'est jamais réalisée.

77. Cette transformation doit être réalisée par un inspecteur général des arts décoratifs. Ce poste est créé le 4 décembre 1881 et confié à Jules Comte, qui enseigne l'histoire de l'art.

78. Le Temps, 23 novembre 1881, p. 2. Cette exposition n'a pas lieu.

79. Après la disparition du ministère, Antonin Proust préside la commission et rédige le rapport : Antonin Proust, Commission d'enquête sur la situation des ouvriers et des industries d'art, Rapport de M. Antonin Proust, Paris, A. Quantin, 1883.

80. Discours à la Chambre des députés, 8 décembre 1881. Sur cette séance et, plus généralement les débats parlementaires occasionnés par la création du ministère, cf. infra.

81. Antonin Proust, « Le salon de 1882 », Gazette des beaux-arts, XXV, juin 1882, p. 548. 
liberté peu conforme à nos habitudes despotiques » affirme en ce sens l'ancien ministre dans son livre de souvenirs ${ }^{82}$. Tout comme le premier, ce principe est socialement fondé : il exprime en effet les goûts et les aspirations des «couches nouvelles » parmi lesquelles se recrutent préférentiellement les producteurs et les consommateurs de l' "art libre » de l'époque ${ }^{83}$. Et tout comme l'unité de l'art, le principe de sa liberté revêt, en plus des dimensions esthétiques et politiques qui s'y attachent, un sens économique : celui, en l'occurrence, de la libre entreprise.

Des actes symboliques viennent signifier cette attitude nouvelle. Des artistes « libres»comme Manet - sont nommés ou promus dans l'ordre de la Légion d'honneur ${ }^{84}$, et Antonin Proust intervient directement pour faire acheter trois toiles de Gustave Courbet par le musée du Louvre ${ }^{85}$. Dans la lignée de celle du Salon, des réformes sont engagées. Celle de l'organisation des musées ${ }^{86}$, avec la création de l'école du Louvre sur le double modèle de l'école pratique des hautes études et de l'école des Chartes ${ }^{87}$ et la fixation des obligations des administrateurs interdisant notamment le cumul des fonctions, favorise la professionnalisation du personnel et son émancipation des milieux érudits et académiques. La refonte du comité consultatif des dons, legs et acquisitions extraordinaires, qui affaiblit le poids des membres de l'Académie des beaux-arts, ouvre la voie à la diversification des collections publiques.

Une autre réforme envisagée - concernant cette fois l'école nationale des beaux-arts - reste quant à elle lettre morte. Reprenant le projet du directeur de l'école Paul Dubois et les préconisations des promoteurs du ministère ${ }^{88}$, le ministre des Arts envisage la suppression des ateliers ${ }^{89}$. Placés sous l'autorité d'un " maître », ceux-ci organisent la transmission des critères de l'excellence artistique. Dès lors que l'administration des beaux-arts procède à la nomination des professeurs des ateliers, l'État intervient de fait — illégitimement selon les tenants de l' " art libre » — dans le choix des normes esthétiques à transmettre — c'est-à-dire en pratique permet la domination des normes académiques dont les «artistes libres»

82. Antonin Proust, Édouard Manet, souvenirs... op. cit., p. 114-115.

83. Qu'un député dénonce l'introduction des artistes indépendants au Salon annuel des beaux-arts comme une « invasion des couches nouvelles » qui menace « les grands noms de l'art français » permet, a contrario, de se convaincre des fondements sociaux de la «liberté de l'art» promue par le gouvernement de Gambetta. Cf. Robert Mitchell, soutenu par les groupes légitimistes, orléanistes et bonapartistes lors de la discussion du projet de réforme du Salon, Chambre des députés, séance du 18 mai 1880, Journal Officiel, 19 mai 1880, p. 5389-5396. 84. Au cours du ministère Proust, Manet, Luc-Olivier Merson, Philippe Gille, Faure, Ferdinand Poise, Albert Lefeuvre, Bracquemond, l'architecte Soty et le forgeron d'art Boulanger sont nommés chevaliers de la Légion d'honneur. Gustave Moreau devient officier, Émile Augier grand officier.

85. Le Combat de cerfs, L'Homme à la ceinture de cuir, L'Homme blessé. Le procédé consiste à faire acquérir les toiles par un collectionneur pour qu'il les cède ensuite à l'État, au cas où le prix des enchères excèderait la somme disponible au budget. On évite ainsi d'avoir à demander un crédit supplémentaire aux Chambres, ce qui occasionnerait un tollé s'agissant de Courbet. D'après ses Souvenirs, Proust aurait voulu faire de même avec Manet, mais ce dernier aurait refusé, ne voulant pas entrer "par petits morceaux » dans les musées nationaux, et revendiquant d'emblée un panneau pour lui seul.

86. Décret du président de la République sur proposition d'Antonin Proust, 24 janvier 1882, Journal Officiel, 26 janvier 1882.

87. Sur la création de l'École du Louvre, cf. Lyne Therrien, L'histoire de l'art en France. Genèse d'une discipline universitaire, Paris, éditions du CTHS, 1998, p. 171 et suivantes.

88. C'est le cas de Leroi dans ses articles de L'Art. Selon Victor Champier : « Le projet de supprimer les ateliers officiels n'avait pas été conçu par M. Antonin Proust ; il était venu à la pensée de tous ceux qui croient que ce n'est pas la mission de l'État d'avoir en art des doctrines et de paraitre les professer. » L'année artistique, op. cit. 89. Les ateliers avaient été créés en 1863, au moment du Salon des refusés, initialement pour donner du travail aux artistes et pour permettre à des artistes non académiques de participer à l'enseignement. Dans l'impossibilité de choisir des artistes acceptés par tous, le gouvernement a le plus souvent pris le parti de la « neutralité », c'està-dire, contrairement aux ambitions initiales des ateliers, nommé des artistes académiques. Cf. Monique Segré, L'École des beaux-arts, XLXe-XXe siècles, Paris, L'Harmattan, « Logiques sociales », 1998, p. 121 et sq. 
entendent s'émanciper. Promulguant un enseignement gratuit, ils constituent par ailleurs selon les réformateurs du ministère une concurrence déloyale pour les ateliers privés, entravant donc la libre entreprise en même temps que la liberté de $1^{\prime}$ art $^{90}{ }^{0}$. Annoncé lors de la première intervention publique d'Antonin Proust comme ministre, à l'occasion de la distribution des récompenses à l'école des beaux-arts le 27 novembre $1881^{91}$, ce projet suscite des débats houleux : « il y eut de la part des intéressés, professeurs et élèves, des protestations aussi vives que celles qui se produisirent au moment de la suppression des corporations », relate ironiquement Antonin Proust ${ }^{92}$. À l'opposition des artistes académiques ${ }^{93}$ s'était en effet ajoutée la mobilisation des élèves des beaux-arts, pour lesquels les ateliers constituaient un moyen d'avoir des cours gratuits ${ }^{94}$

\section{L'échec d'une institutionnalisation conflictuelle}

Très vite, les conditions qui l'avaient rendu possible se retournent contre le ministère. Tout d'abord, les réformes de Gambetta visant à renforcer l'autorité politique sur l'administration centrale n'ont pu que susciter les réticences et résistances de la «bureaucratie oiseuse » contre laquelle elles étaient dirigées. La redéfinition des territoires bureaucratiques engagée à cette occasion n'a pas non plus été sans réactiver les concurrences entre administrations. Le nouveau ministère des Arts, dont les principaux services proviennent du ministère de l'Instruction publique, a ainsi subi les attaques du personnel de ce département. De même des fonctionnaires du ministère du Commerce qui revendiquent — tout comme ceux de l'Instruction publique - la compétence de l'enseignement technique ${ }^{95}$, ou encore du service des Cultes, dont les membres s'opposent au transfert des édifices diocésains et des cathédrales $^{96}$. Dès lors que les arts industriels constituaient un axe majeur du ministère des Arts, ce dernier se trouvait également pris dans les concurrences multiples pour le traitement de la question du travail qui opposent alors notamment le ministère de l'Intérieur à celui du Commerce $^{97}$.

Ensuite, le soutien des milieux des arts décoratifs, celui des artistes « libres », l'orientation qui s'ensuit dans l'organisation et les pratiques du ministère des Arts pouvaient difficilement ne

90. Antonin Proust, Edouard Manet, souvenirs..., op. cit., p. 116.

91. Le Temps, 28 novembre 1881.

92. Antonin Proust, L'Art sous la République, op. cit., p. 54.

93. L'artiste « juste milieu » Ferdinand Humbert, qui a son atelier à l'École des beaux-arts, dit en substance la même chose que ses collègues Alexandre Cabanel, Jean-Léon Gérôme (académiciens, et plus âgés que lui) : « C'est dans les ateliers que se conservent les traditions de l'École. Dans un temps où des théories indépendantes et anarchiques se manifestent dans l'art, où les jeunes gens ne demanderaient pas mieux que de s'affranchir des règles jusqu'ici respectées sans savoir par quoi les remplacer, la suppression des ateliers serait funeste. »Cité in Monique Segré, L'École des beaux-arts, op. cit., p. 127.

94. L'artiste, 1881 ; Le Journal des arts, 13 décembre 1881. Le Figaro publie quant à lui des articles favorables à la suppression des ateliers.

95. Sur cette question, voir Jean-Pierre Briand, Jean-Michel Chapoulie, Les collèges du peuple : l'enseignement primaire supérieur et le développement de la scolarisation prolongée sous la Troisième République, Paris, INRP-CNRS-ENS Fontenay-Saint-Cloud, 1992.

96. Cf. Jean-Michel Leniaud, L'Administration des cultes pendant la période concordataire, Paris, Nouvelles éditions latines, 1988.

97. Sur cette question, et notamment sur les recompositions du ministère du Commerce dans les décennies 1880$1890, \mathrm{cf}$. Didier Renard, Initiative des politiques et contrôle des dispositifs décentralisés. La protection sociale et l'État sous la Troisième République, 1885-1935, Cachan, GAPP, rapport pour la MIRE, 2000, p. 47 et suivantes. 
pas susciter l'hostilité des tenants de l'académisme dont l'influence, bien que déclinante, reste forte dans les institutions artistiques ${ }^{98}$. L'article que publie l'académicien conservateur Henry Houssaye dans la Revue des deux mondes donne un bon exemple de ces réactions hostiles ${ }^{99}$. L' " unité des arts » conduit selon lui à un dangereux bouleversement des hiérarchies du Beau. Et l'académicien d'ironiser sur la nouvelle catégorisation promue par le ministère des Arts :

«Que les écoles et manufactures d'art industriel et décoratif soient rattachées au développement des arts, cela est fort logique. Mais quels rapports secrets existent entre l'art et la mécanique, la filature, le tissage, la chaudronnerie, la construction des machines, la chimie agricole, la tenue des livres ? Quelle compétence auront les inspecteurs des beauxarts pour juger l'enseignement donné à l'école d'horlogerie de Cluses ou à l'école de tissage de Lille ? Voilà des innovations sans motifs, voilà où commence le bouleversement. L'art n'est pour rien dans un métier à tisser, attendu que celui qui l'a fait ne s'est nullement inquiété de sa forme plastique. Où il n'y a pas souci de la forme, il n'y a pas d'art. »100

Selon lui, la «liberté de l'art »n'est en réalité qu' « un mot à effet», qui désigne " les nouvelles écoles d'ignorance et de vulgarité donnant assaut aux traditions des maîtres ».
« Rapprochez l'entrée au Louvre de huit ou neuf tableaux de Courbet de la suppression des ateliers officiels, des déclarations du ministre sur l'indépendance de l'art, des discussions passionnées à propos de l'école de Rome et de la décoration de M. Manet, et vous comprendrez qu'il y a menace de rupture entre l'administration et le grand art. On va combattre les idées de noblesse, de grâce, de grandeur et de beauté au profit d'un monstrueux idéal de brutalité et de bassesse. La liberté de l'art, ce sont les nouvelles écoles d'ignorance et de vulgarité donnant assaut aux traditions des maîtres; c'est la Vénus hottentote qui dit : ôte-toi de là ! à la Vénus grecque. »101

Viennent ensuite les conditions politiques de création du ministère qui, elles aussi, se retournent contre lui. Aux hostilités bureaucratiques et académiques s'ajoutent voire se combinent en effet, dès novembre 1881, des oppositions au sein du champ politique. C'est surtout à la Chambre que les débats sont vifs, après le dépôt par le député Paul Leroy membre du groupe de l'Union républicaine - du rapport pour la demande de crédits supplémentaires pour les nouveaux ministères, le 24 novembre 1881, et lors de la discussion de ce rapport en présence des ministres, le 8 décembre. Des députés de droite, et en particulier Franck Chauveau, interviennent alors pour critiquer les changements apportés à la division du travail gouvernemental.

Ces changements et les oppositions qu'ils suscitent s'inscrivent alors dans les concurrences entre le Parlement et un gouvernement qui, au regard de la configuration habituelle du jeu politique de l'époque, s'en est émancipé. Les réformes engagées doivent préserver l'administration de « la politique du scrutin d'arrondissement », ces pressions et sollicitations parlementaires que dénonce Gambetta ${ }^{102}$. Si la création de nouveaux ministères a pu être

98. Cf. Albert Boime, The Academy and French Painting... op. cit.

99. Fils d'Arsène Houssaye (1815-1896), littérateur et administrateur de la Comédie française de 1849 à 1856, Henry Houssaye, né en 1848, a entre autres écrit des ouvrages historiques sur Napoléon. Henry Houssaye, « Le ministère des Arts », Revue des deux mondes, ler février 1882, p. 613-627.

100. Ibid., p. 615-616.

101. Ibid., p. 626.

102. Cf. Joseph Reinach, Le ministère Gambetta, op. cit., p. 337. L'intervention des parlementaires auprès de l'administration des beaux-arts fait l'objet de très fréquentes dénonciations de la part de critiques et d'artistes. " L'assaut du sous-secrétariat d'État aux Beaux-Arts au profit de toutes les médiocrités de leurs arrondissements a été incessant de la part de presque tous les députés ", écrit ainsi Paul Leroi. (" Le sous-secrétariat des BeauxArts», art. cit., p. 231.) 
dénoncée comme un " crime de lèse-parlementarisme », pour reprendre l'expression ironique d'Antonin Proust ${ }^{103}$, c'est aussi qu'elle a été conçue et décidée au seul niveau gouvernemental. Lors des débats du 30 novembre, les membres de la commission parlementaire regrettent ainsi que la disparition de la constitution de 1848 fasse qu'il ne soit plus nécessaire de voter une loi pour la création d'un ministère, et affirment le principe qu'à l'avenir aucun ministère ne devra être créé sans l'aval des parlementaires ${ }^{104}$. Au cours de la séance du 8 décembre, Franck Chauveau cherche à démontrer les contradictions du gouvernement en faisant référence aux débats du second Empire. Il cite en particulier les arguments de ce qu'il désigne comme « la gauche » de l'époque, opposée à la création du ministère des Beaux-Arts en 1870, et rappelle à l'inverse ceux des partisans de l'Empire, en les rapprochant de ceux utilisés par le député Leroy pour justifier l'édification du ministère des Arts. Il mobilise également les constitutions et textes révolutionnaires qui prévoient le rôle de l'assemblée pour la création des ministères.

« Eh bien je dis qu'il n'est pas possible, sous un gouvernement républicain, d'admettre de pareils procédés ; je dis qu'il n'est pas possible de créer ainsi des fonctions, dont l'utilité est contestable, sans avoir consulté le Parlement, et de les présenter ensuite à sa ratification, quand la dépense est faite et qu'il peut à peine se soustraire à la nécessité de la voter. ${ }^{105}$

Si elles sont critiquées, c'est qu'au-delà du procédé utilisé par le gouvernement pour les accomplir, "ces innovations paraissent être, non pas la réalisation de vues passagères ou de modifications isolées, mais le premier symptôme d'un système gouvernemental, l'essai d'une politique nouvelle »106. C'est en particulier le cas du ministère des Arts, " la création favorite » des décrets du 14 novembre, " car c'est elle qui s'est le plus enrichie des dépouilles des autres départements ministériels »107. Ce ministère est de fait «la création la plus contestée de toutes » 108 , et obtient plus difficilement que les autres le soutien des députés ${ }^{109}$.

La création du ministère des Arts soulève principalement trois problèmes. Le premier, dans le prolongement des critiques formulées par les agents du pôle académique du champ artistique, tient aux regroupements opérés sur la base d'une définition élargie des arts. Franck Chauveau dénonce ainsi le fait

\begin{abstract}
" d'annexer à ce service des éléments hétérogènes et des attributions disparates que le caprice d'un jour, non la nature des choses, a réunies, et que l'expérience du lendemain séparera infailliblement. [...]Le ministère des Arts, poursuit-il, est devenu une sorte de mosaïque ; on y a fait entrer toutes sortes d'attributions nouvelles ; mais il est difficile de comprendre quelle vue d'ensemble a présidé à cette organisation. » Il cite en particulier les bâtiments civils, dont certains n'ont «rien d'artistique», les édifices diocésains et paroissiaux, pour ce qui concerne les grosses réparations (l'entretien courant restant aux
\end{abstract}

103. Dans un article paru après la chute du cabinet Gambetta dans la Revue politique et littéraire, cité dans Le Temps du 21 février 1882.

104. Le Temps, ler décembre 1881.

105. Chambre des députés, séance du 8 décembre 1881, Journal officiel des débats, 9 décembre 1881.

106. Franck Chauveau, Chambre des députés, séance du 8 décembre 1881, Journal officiel des débats, 9 décembre 1881.

107. Ibid.

108. « Le ministre des Arts devant le Parlement », Le journal des arts, 13 décembre 1881.

109. C'est, en plus des débats, ce que fait apparaitre la comparaison du vote des crédits pour la création des deux ministères des Arts et de l'Agriculture : respectivement quatre cent quarante-sept votants dont trois cent trentehuit pour et cent neuf contre, et quatre cent soixante-cinq votants dont trois cent soixante-dix pour et quatrevingt-quinze contre, soit une différence au détriment du ministère des Arts de quatorze votes hostiles, dix-huit abstentions et trente-deux voix favorables. Cette différence s'explique en partie par le ralliement du groupe de Clemenceau au vote hostile de la droite. 
Cultes); les écoles professionnelles dont certaines sont «scientifiques » plus qu' « artistiques ».

Il s'agit ensuite de la définition des fonctions gouvernementales. Les nouveaux départements, et en particulier celui des Arts, seraient « inutiles », et auraient "été faits non pas pour créer des ministères, mais bien pour faire des ministres » 110 . Lorsque, le 29 novembre, Gambetta et les nouveaux ministres se rendent à la commission des crédits supplémentaires pour justifier les récentes créations, les députés Louis Legrand et Ribot défendent quant à eux le territoire administratif de l'Instruction publique, en pressentant des conflits de compétence avec le ministère des Arts $^{111}$. Quand bien même la réorganisation des services est jugée positive, c'est la promotion au rang de ministère qui fait l'objet de critiques, notamment en raison des frais supplémentaires occasionnés par le traitement du ministre et des membres de son cabinet ${ }^{112}$.

À partir de ces réserves, les parlementaires hostiles soulèvent un troisième problème, dénonçant au travers du ministère des Arts les risques plus généraux de l'étatisation. L'étatisation, c'est d'abord le fait que le gouvernement s'immisce " dans un ordre de choses qui appartient à l'initiative privée "), celui de l'art mais aussi en l'occurrence de l'industrie'113. «L'ingérence du gouvernement dans toutes les sphères de l'activité individuelle » risque en matière artistique de conduire à « un art d'État »114 en contradiction avec la « libération » des arts annoncée par le nouveau ministre ${ }^{115}$. L'étatisation, c'est enfin l'augmentation inéluctable des dépenses publiques, que la Chambre ne pourra pas contrôler, et le « fonctionnarisme » qui s'ensuit, contrairement à la réduction de «l'armée encombrante des employés de l'administration » annoncée par Gambetta, et avec au final des incidences négatives sur le développement de l'art que la création du ministère est censée permettre.

« L'importance du service des beaux-arts justifiait-elle cette création ? Eh bien, messieurs, je ne le pense pas. Je ne parle pas, bien entendu, de l'importance intellectuelle ou morale du service; je parle de son importance matérielle, du plus ou moins grand nombre d'employés, de la complication des services, de tout ce qui, en un mot, peut justifier la création d'un ministère. Certes, je suis, autant que personne, partisan de l'intervention tutélaire et discrète de l'État dans les choses de l'art; pas plus que personne ici, je ne voudrais découronner la France en diminuant cette auréole de gloire que lui font ses littérateurs et ses artistes; mais je me demande ce que tout cela a de commun avec l'accroissement du nombre des fonctionnaires. Croyez-vous, en créant des fonctionnaires, créer du même coup des peintres et des sculpteurs? Croyez-vous ajouter au génie des artistes en élevant les titres et en augmentant les appointements de vos employés ? La mission de l'État, dans cet ordre de choses, est naturellement noble et grande [...] mais ce n'est point là une question de ministère, et une simple direction suffit parfaitement à cette protection et à cet encouragement éclairé. »116

L'on comprend qu'une fois retrouvées les conditions habituelles du jeu politique parlementaire et terminée la parenthèse $d u$ " grand ministère », les réserves exprimées lors de

110. Chambre des députés, séance du 8 décembre 1881, Journal officiel des débats, 9 décembre 1881.

111. Le Temps, 30 novembre 1881.

112. C'est notamment le cas de Lambert de Sainte Croix, sénateur de droite, pour qui un secrétariat d'État voire une direction administrative auraient suffi. Le Temps, 17 décembre 1881. Les réserves opposées à la création des ministères sont nettement moins importantes au Sénat qu'à la Chambre des députés. Les sénateurs votent à une large majorité et après un débat nettement moins nourri les crédits supplémentaires liés à ces créations.

113. Louis Legrand, cité in Le Temps, 30 novembre 1881.

114. Beauquier, Chambre des députés, séance du 8 décembre 1881, loc. cit.

115. Chauveau, ibid.

116. Idem. 
la création du ministère des Arts deviennent des motifs pour sa suppression. Plus encore, la création de ce ministère symbolisant le rapport de forces politique inédit qui l'avait rendu possible, son démantèlement, avec la formation du cabinet Freycinet, symbolise le retour au status quo ante. "Les événements parlementaires ont mis à néant notre entreprise », regrette Antonin Proust ${ }^{117}$. Autrement dit, le jeu politique a défait ce qu'un état particulier du jeu politique avait permis de faire.

« Si [la politique] a amené au grand jour des discussions tumultueuses, certains problèmes et certaines questions d'administration pour l'examen desquels le dilettantisme bienveillant ne suffit pas, il est à craindre qu'elle ait compromis pour un temps les réformes longuement espérées par les hommes véritablement compétents en ces matières délicates. [...] Défendue ou attaquée, comme elle l'a été durant un mois, l'idée d'un ministère des Arts n'a pu se présenter à l'esprit du public qu'à travers les passions politiques; or ce ne sont pas ces passions-là qui permettent de bien voir et qui font les bonnes besognes. [...] Ce qui reste démontré, à l'heure qu'il est, c'est qu'on ne possédera en France une véritable et utile administration des arts que lorsqu'on sera parvenu à la soustraire aux fluctuations, aux accidents journaliers de la politique et aux incessants changements de personne. »118

Ces « événements parlementaires » dont le démantèlement du ministère des Arts procède directement ont d'autant plus de poids qu'ils s'ajoutent et se combinent à des oppositions et des concurrences établies dans d'autres espaces sociaux - en particulier au sein des champs bureaucratique et artistique (du côté du pôle académique).

Au lendemain de la chute de son gouvernement, Gambetta prédit à Proust : " Vous avez été, mon cher ami, [...] le premier ministre des Arts. Vous serez vraisemblablement le dernier ${ }^{119}$. Et de fait, ce ministère des Arts que les promoteurs ultérieurs d'une politique artistique ont régulièrement cité en exemple puis progressivement oublié n'a jamais été reconstitué. Quinze ans après sa disparition, l'un des principaux d'entre regrette que

« Les Chambres et l'opinion ne semblent pas disposées à le rétablir. [...] Ce n'est pas là une création d'aujourd'hui ni de demain. La République attendra longtemps avant de reprendre l'idée de Gambetta. »120

C'est qu'au-delà des causes immédiates de sa mise à l'écart, cette "idée » n'a plus trouvé les conditions sociales de sa réalisation. De fait, si l'on pouvait envisager une " union de l'art et de l'industrie » sous l'égide du gouvernement au début des années 1880, l'autonomisation croissante du champ artistique à l'égard des champs économique et politique rend cette triple alliance de moins en moins pensable ${ }^{121}$.

Prenant acte de cette étatisation manquée, Gambetta, Proust et leurs soutiens escomptaient la reprise du projet d' ' union des arts » en dehors des institutions gouvernementales. Même à ce niveau, le "programme » du ministère Proust n'a cependant que peu été poursuivi. Ce fût partiellement le cas, avec la fusion de l'Union des beaux-arts appliqués à l'industrie et de la Société du musée des arts décoratifs au sein de l'Union centrale des arts décoratifs, dont

117. Antonin Proust, L'Art sous la République, op. cit., p. II. Voir aussi les remarques dont il émaille ses articles sur « Le Salon de 1882 », Gazette des beaux-arts, XXV, juin 1882, p. 533-554 et XXVI, août 1882, p. 145-160.

118. Victor Champier, L'année artistique, op. cit., successivement p. 1 et p. 13-14.

119. Antonin Proust, L'Art sous la République, op. cit., p. IV.

120. Gustave Larroumet, L'Art et l'État en France, Paris, Hachette, 1895, p. 305-306.

121. Sur l'histoire de l'opposition entre « l'art et l'argent », voir Pierre Bourdieu, Les règles de l'art, Paris, Seuil, 1992. Sur l'opposition entre «l'art et l'État», on se permet de renvoyer à Vincent Dubois, « L'art et l'État au début de la IIIe République », art. cit. ; La politique culturelle..., op. cit. 
Antonin Proust devient président après son départ du gouvernement ${ }^{122}$. De nouvelles conceptions de l'art, comme l' « art social », promu par Roger Marx à la fin du XIXe siècle, en reprendront partiellement l'héritage; et les arts décoratifs ont connu le développement que l'on sait dans l'entre-deux-guerres.

Mais, pas plus que l'objectivation d'une politique artistique — il faudra de fait attendre la création du ministère des Affaires culturelles en 1959 pour que soit revendiquée et institutionnalisée l'intervention étatique dans ce domaine - le ministère des Arts et ses suites ne donnent lieu à la révolution annoncée dans les manières de considérer l'art. C'est sans doute qu'au-delà des péripéties politiques qui en ont directement marqué l'abandon, cette prophétie qui se voulait créatrice était insuffisamment fondée dans la réalité. Ou, pour le dire autrement, que pour avoir trop anticipé sur ce qui n'était peut-être pas vraiment en train d'advenir - la redéfinition des classements et hiérarchies esthétiques par la « réunion des arts » - , la taxinomie du ministère des Arts n'a pu, faute d'avoir été l' « accoucheuse de l'histoire », pas même en être « l'officier d'état civil » 123 .

Vincent Dubois

122. Debora L. Silverman, L'art nouveau en France, op. cit., p. 127. L'Union est par la suite reconnue comme établissement d'utilité publique. Voir aussi Évelyne Possémé, « La politique de reproduction à l'Union centrale des arts décoratifs au XIXe siècle », in Chantal Georgel (dir.), La jeunesse des musées, Paris, RMN, 1994, p. 77 82.

123. On emprunte ces expressions à Pierre Bourdieu, « La force du droit. Éléments pour une sociologie du champ juridique », Actes de la recherche en sciences sociales, 64, décembre 1986, p. 13. 


\section{Rapport au Président de la République pour la création du ministère des Arts Léon Gambetta, Journal Officiel, 17 novembre 1881}

Monsieur le Président,

Aucun de nos services publics n'a subi plus de modifications que le service des beaux-arts, soit dans ses attributions, soit dans ses rapports avec les autres services. Sous l'Ancien Régime, il était compris dans l'administration des Bâtiments Royaux et il a longtemps fait partie de l'administration des Domaines. En 1792 il a été rattaché au ministère de l'Intérieur, où il fut divisé en trois bureaux puis en trois sections. Le Premier Empire le réduisit aux proportions d'un seul bureau. La Restauration en fit une Division. La Monarchie de 1830 l'attribua d'abord au ministère du Commerce et des Travaux Publics et le partagea plus tard entre le ministère de l'Intérieur et le ministère de l'Instruction Publique. En 1848, le service des beaux-arts fut placé dans la direction des Musées nationaux. Le Second Empire le fit entrer en 1853 au ministère d'État et le plaça douze ans après dans les attributions de la Maison de l'Empereur la direction des Bâtiments Civils et la direction des Sciences et Lettres. Le 28 août 1870, le ministère spécial des Beaux-Arts fut supprimé et ses attributions transportées au ministère de l'Instruction Publique qui, après le 4 septembre, abandonna au ministère des Travaux Publics la direction des Bâtiments Civils.

Dès les premiers mois de la réunion de l'Assemblée nationale, $\mathrm{M}$. Charton, dans un rapport fait au nom de la commission des services administratifs, appela l'attention du gouvernement sur les inconvénients de cette incessante mobilité du service des beaux-arts. Et le ler février 1878, M. Bardoux, ministre de l'Instruction Publique et des beaux-arts, visant le rapport de M. Charton, forma une commission chargée d'étudier les modifications qui pourraient donner plus de cohésion et de solidité à l'organisation de la deuxième section de son département. L'institution d'un sous-secrétariat, ayant dans ses attributions spéciales le service des beauxarts, permit en 1879 de réaliser quelques unes des réformes qu'avait proposées la commission de 1878, mais la réunion des services qui intéressent l'enseignement de l'art à tous les degrés et dans toutes ses applications n'a pas été opérée.

Cependant les nations étrangères ont depuis bientôt trente ans multiplié les institutions destinées à favoriser cet enseignement, et nous avons pu constater à l'Exposition universelle de 1878, combien ont été rapides, grâce à la prévoyance de leurs gouvernements, les progrès accomplis dans les industries d'art par des peuples qui n'étaient encore naguère que nos imitateurs et qui sont devenus nos rivaux.

La constatation de cet état de choses a donné lieu en France à un véritable mouvement d'opinion en faveur d'institutions qui ont donné partout de si prompts et de si précieux résultats. Après avoir rappelé que c'est la France qui a, la première, ouvert les conservatoires et les écoles d'arts et métiers, que c'est à l'initiative des hommes de la Révolution que sont dus les musées et les corps spéciaux qui assurent l'alliance de l'art et de l'industrie, on a justement fait remarquer que, dans nos Sociétés modernes, il ne suffit pas de développer le goût et la culture des beaux-arts, mais que l'assistance que l'art peut donner à l'industrie a une importance considérable au point de vue économique et social, puisqu'elle transforme les conditions du travail et exerce une influence décisive sur les forces productives d'une nation.

Pour développer ce large enseignement des principes généraux de l'art que réclament nos grandes industries, pour fortifier l'enseignement technique qui ne leur est pas moins nécessaire, j'ai l'honneur, monsieur le Président, de vous proposer la création d'un ministère des Arts.

Ce ministère aurait dans ses attributions l'administration actuelle des beaux-arts, la direction des Bâtiments civils et la construction des édifices diocésains et cathédrales, services qui, par leur caractère se rattachent naturellement au service des beaux-arts. Il aurait, en outre, la direction de l'enseignement technique (Conservatoire et écoles d'Arts et Métiers) qui est actuellement dans les attributions du ministère de l'Agriculture et du Commerce, et enfin l'inspection et la surveillance de l'enseignement du dessin dans les Ecoles normales d'instituteurs et d'institutrices, dans les écoles primaires, dans les collèges et lycées.

Je m'empresse d'ajouter, monsieur le Président, que la création que j'ai l'honneur de vous proposer ne me parait pas devoir amener au budget de l'État l'accroissement de dépense que justifierait d'ailleurs le grand intérêt qui s'y attache. J'estime, en effet, que pour faire une œuvre utile, vraiment féconde et réellement conforme à nos traditions, à nos aptitudes, à nos tendances, le ministères des Arts n'a pas besoin de multiplier les établissements d'État toujours coûteux et souvent stériles. Mon sentiment est qu'il doit s'attacher à associer l'action du pouvoir central, dans la mesure où celle-ci a le devoir de se produire à l'action des corps ou associations déjà constitués, qu'il lui faut faire appel aux départements, aux communes, aux Chambres de Commerce, aux comités consultatifs des Arts et Manufactures, aux syndicats professionnels, qui attendent tous du gouvernement de la République le concours que ce gouvernement est tenu plus que tout autre de prêter aux efforts de notre démocratie laborieuse. 
J'espère, monsieur le Président, que vous voudrez bien accueillir favorablement la proposition que j'ai l'honneur de soumettre à votre haute approbation.

Veuillez agréer, monsieur le Président, l'hommage de mon profond respect.

Le Président du Conseil, ministre des Affaires étrangères, Léon GAMBETTA 